\title{
Microscopic structure and distribution of various elements in the eggshell of the Black-tailed Gull, Larus crassirostris, as revealed by scanning and transmission electron microscopy and X-ray compositional microanalysis
}

\author{
Akira CHIBA $^{\#}$ \\ Department of Biology, Nippon Dental University School of Dentistry at Niigata, Niigata 951-8580, Japan
}

\begin{tabular}{c}
\hline ORNITHOLOGICAL \\
SCIENCE \\
$\begin{array}{c}\text { @ The Ornithological Society } \\
\text { of Japan } 2004\end{array}$ \\
\hline
\end{tabular}
structures and have long been objects of studies in poultry science, ornithology, and general biology. A considerable amount of knowledge on the morphology and physiology of eggshells has hitherto been accumulated and reviewed (Romanoff \& Romanoff 1949; Gilbert 1979). The greater part of our knowledge of eggshell structure was obtained from studies on the domestic hen Gallus gallus (Bellairs \& Boyde 1969; Fujii \& Tamura 1969; Tung \& Richard 1972; Tan et al. 1992; Dennis et al. 1996; Fraser et al. 1999). However, there is a growing body of data from the birds of various taxonomic groups (Tyler 1964, 1965, 1966, 1969; Becking 1975; Tullet et al. 1976; Tullet 1984; Mikhailov 1995a, b). These studies have contributed not only to comparative morphology of

(Received 10 February 2004; Accepted 13 May 2004)

\# E-mail: chiba@ngt.ndu.ac.jp the eggshells, but also to the respiratory physiology of the developing embryos (Paganelli et al. 1975; Rahn \& Paganelli 1981; Tullet 1984) and consideration of avian systematics (Mikhailov 1995b). These studies have also revealed variations in the microscopic structures of eggshells and discrepancies in terminology used to describe them (Gilbert 1979; Mikhailov 1995a; Dennis et al. 1996). For a better understanding of the structural details and functional properties of avian eggshells, comprehensive studies conducted with the aid of different techniques are needed. To our knowledge, however, such studies are scanty and seem to be limited to the eggshell of domestic fowl (Tan et al. 1992; Dennis et al. 1996; Fraser et al. 1998; Richards et al. 2000).

The present study was conducted to expand our knowledge of the microscopic structure and the distribution of elements or minerals in the eggshells of wild Japanese birds by the use of modern techniques. 
For this purpose, the eggshell of the Black-tailed Gull Larus crassirostris was selected for sampling and investigation.

\section{MATERIALS AND METHODS}

\section{1) Materials}

Four fresh, but slightly broken, Black-tailed Gull eggs were used in this study. They were collected (by permission of the Ministry of Environment) from two abandoned nests, during a field survey of breeding colonies on the coast of the Japan Sea in the northern district of Niigata Prefecture. The nest abandonment and egg damage seemed to have been caused by mischievous intruders. The eggs were opened in the laboratory, and their contents were carefully examined and removed. The shells were then processed for microscopic observation. The eggs used were diagnosed to have been fertile and in an early stage of normal embryonic development.

\section{2) Scanning electron microscopy}

For SEM, parts of the eggshells were washed in tap water, rinsed with distilled water, cut into small pieces (about $5 \times 5 \mathrm{~mm}$ ), and dried for $48 \mathrm{hr}$ at $38^{\circ} \mathrm{C}$ in an oven. Then, they were mounted on an aluminum stub, sputter-coated with platinum-palladium, and examined with a Hitachi H-800 scanning electron microscope. The rest of the eggshells were incubated overnight in a $1 \%$ solution of potassium hydroxide at $38^{\circ} \mathrm{C}$ to remove organic compounds from the shells, washed in tap water, rinsed with distilled water, and dried in the oven. Then, they were similarly processed and examined for comparison.

\section{3) Light and transmission electron microscopy}

For TEM, pieces of the shells were appropriately trimmed, washed briefly in tap water, rinsed with $0.1 \mathrm{M}$ phosphate-buffered saline $(0.01 \mathrm{M}$ sodium phosphate, $0.15 \mathrm{M}$ sodium chloride, $\mathrm{pH}$ 7.2) and fixed in a solution of $0.1 \mathrm{M}$ sodium cacodylate containing $1 \%$ glutaraldehyde and $0.1 \mathrm{M}$ sodium ethylenedimine-tetraacetate (EDTA), pH 7.6. For fixation and decalcification, the samples were placed in conical tubes and continuously shaken with an electric shaker. After $24 \mathrm{hr}$, the fixative was replaced with freshly prepared $0.1 \mathrm{M}$ sodium cacodylate containing $0.1 \mathrm{M}$ EDTA. The samples were then incubated in this medium overnight, with gentle shaking, to remove any remaining calcium. Next, they were washed three times in $0.1 \mathrm{M}$ sodium cacodylate, post- fixed for $1 \mathrm{hr}$ in $0.1 \mathrm{M}$ sodium cacodylate containing $1 \%$ osmium tetroxide, and washed once in $0.1 \mathrm{M}$ sodium cacodylate and twice in distilled water. The samples were then dehydrated by passage through a graded ethanol series and embedded in Spurr resin. Semi-thin sections were stained with toluidine blue in borax and observed under a light microscope. Ultrathin sections were cut with a diamond knife, stained with uranyl acetate and lead citrate, and viewed with a JEOL 1200 EX electron microscope.

\section{4) X-ray compositional microanalysis}

Pieces of the shells were embedded in epoxy resin and a radial face was polished with successively finer grades of diamond paste (final grade, $1 \mu \mathrm{m}$ ). A final polish was given with aluminum oxide $(0.3 \mu \mathrm{m}$ grade) as a paste in velvet supported by a plate glass. The polished surface was coated in vacuo with gold (ca. $30 \mathrm{~nm}$ ) and examined with a JEOL JXA-50A electron probe microanalyzer. The electron beam (accelerating voltage, $20 \mathrm{kV}$; beam current, $0.009 \mu \mathrm{A}$ ) was scanned along a line across the polished radial face, beginning at the shell membrane, continuing across the tip of a cone, and terminating at the plastic at the outer edge of the shell. The results on $\mathrm{Ca}, \mathrm{Mg}$, and $\mathrm{P}$ were recorded on a chart moving at a known speed. No examination was made of other elements, such as $\mathrm{O}$ or $\mathrm{S}$.

\section{RESULTS}

1) Scanning electron microscopy

SEM examination of the Black-tailed Gull eggshell showed a complex architecture that differed within each of the major zones of the eggshell: the shell membrane (SM), the mammillary zone (MZ), the palisade region (PR), and the cuticle layer (CL; Fig. 1A). The SM, the innermost zone of the eggshell, was $139.7 \pm 2.4 \mu \mathrm{m}(\mathrm{mean} \pm \mathrm{SD}, \mathrm{N}=4)$ thick and consisted of three layers, i.e. the limiting membrane (LM), inner SM, and outer SM. The LM that contacts directly with the albumin of intact eggs appeared as a thin film and could be partly detached from the inner SM by utilizing a pair of forceps (Fig. 1B). The inner $\mathrm{SM}, 51.4 \pm 5.3 \mu \mathrm{m}(\mathrm{N}=4)$ thick, was composed of fibers that ran parallel to the eggshell surface and crossed each other at different angles. The individual fibers measured $1.2 \pm 0.2 \mu \mathrm{m}(\mathrm{N}=10)$ in mean diameter and had minute granular processes on its surface. The outer SM, $90.6 \pm 2.3 \mu \mathrm{m}(\mathrm{N}=10)$, was also composed of similar fibers, although each individual fiber 


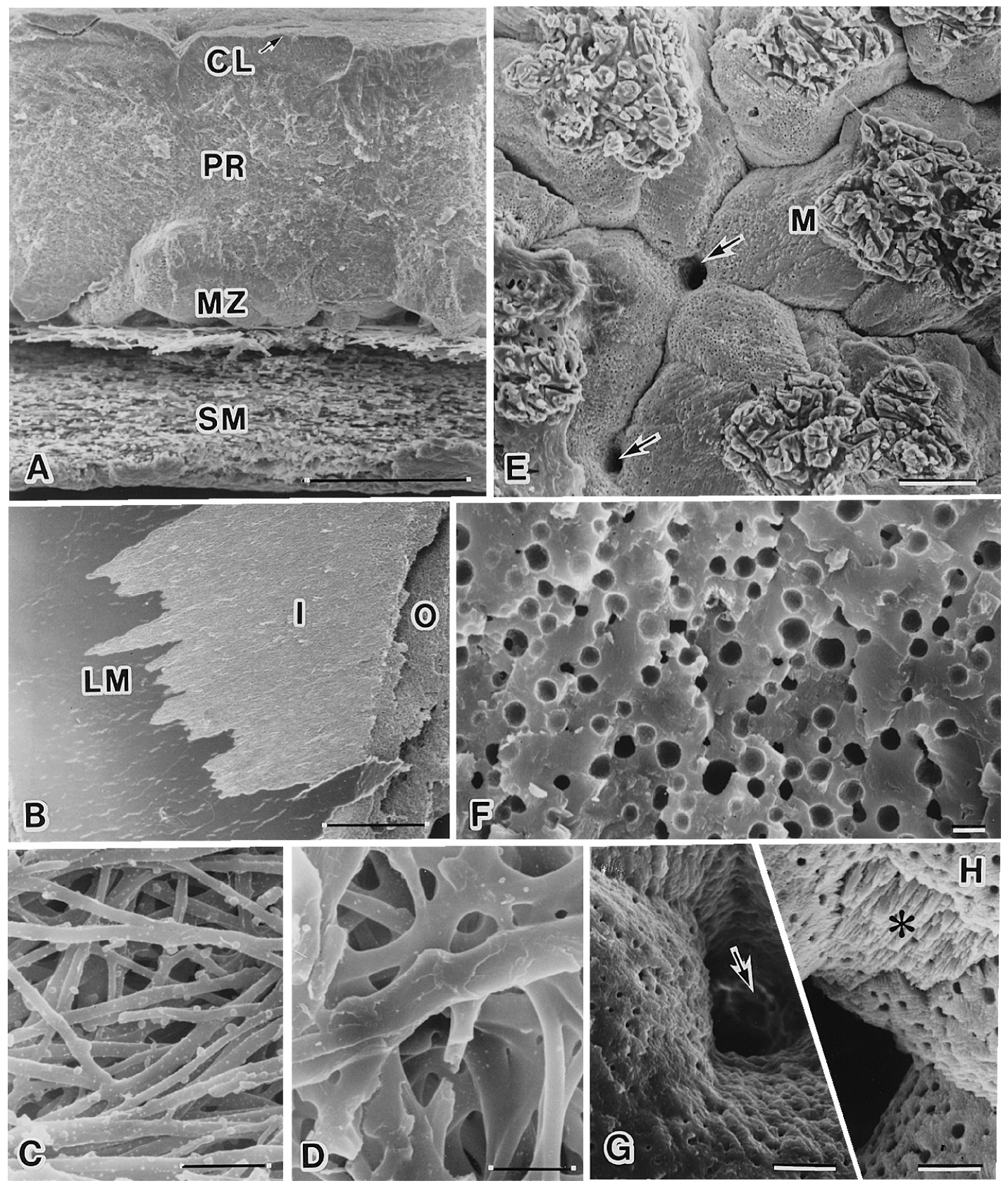

Fig. 1. SEM micrograph of an eggshell of the Black-tailed Gull Larus crassirostris, showing its inner and surface fine structures. (A) low-power view of radially fractured face of the eggshell, showing the cuticle layer (CL indistinct due to low-power view), the shell membrane (SM), mammillary zone (MZ), and palisade region (PR). (B) the shell membrane consisting of the limiting membrane (LM), and inner (I) and outer (O) SMs. (C \& D) details of the inner (C) and outer (D) SMs composed of thin fibers. (E) inner face of the MZ showing the mammillare (M) and internal opening (arrows) of the pore canal after maceration with potassium hydroxide. (F) closer view of the PR, showing its spongy feature owing to the numerous vesicles embedded in the calcified matrix. (G \& $H)$ closer view of the external opening of the pore canal, with $(\mathrm{H})$ and without $(\mathrm{G})$ maceration. In the macerated specimen $(\mathrm{H})$, the densely mineralized PR with crystal structure (asterisk), is disclosed and shows a rough surface due to the removal of the CL from the shell. In contrast, the non-macerated specimen (G) shows a rather smooth surface due to the presence of the cuticle layer. Organic or cuticle substance (arrow in G) is visible in the pore canal. Scale bar $=100 \mu \mathrm{m}$ (A \& B), $50 \mu \mathrm{m}$ (E), $5 \mu \mathrm{m}$ (C, D, G, \& H), $1 \mu \mathrm{m}$ (F). 
of the outer SM was thicker, $3.8 \pm 0.6 \mu \mathrm{m}(\mathrm{N}=10)$ in diameter, than the fibers of the inner SM and had a smooth surface (Figs. 1C, D). The border between the inner and outer SMs was usually indistinct. The MZ occupied about $25-30 \%$ of the thickness of the mineralized (calcified) portion of the eggshell, and was $79.8 \pm 2.4 \mu \mathrm{m}(\mathrm{N}=4)$ thick. It consisted of numerous conical knobs, the mammillare, the apices of which were embedded in the outer SM and, via the apices, fibers from the outer SM were connected with the mammillare (Fig. 1A). This feature of the apices was clearly shown as irregular processes and grooves in the mammillare when the shell had been macerated with potassium hydroxide solution (Fig. 1E). The PR external to the MZ had a spongy feature, being endowed with numerous vesicles ranging from 0.5 to $1.3 \mu \mathrm{m}(0.9 \pm 0.1 \mu \mathrm{m} ; \mathrm{N}=4)$ in diameter (Fig. 1F). Sometimes, two or more adjacent vesicles were connected to each other. Occasionally the PR showed a squamatic pattern. The external part of the PR was distinguished from the major (spongy) part of the PR; there were few vesicles, and the calcified matrix appeared to be very compact, showing an appearance of denser mineralization (Fig. 1A). This part was comparable to the external zone or surface crystal layer recognized in other species. The CL, the outermost layer of the shell, was thin $(4.1 \pm 0.4 \mu \mathrm{m}$ thick; $\mathrm{N}=4)$ and contained occasional vesicles or minute pores. It was devoid of any microglobular structures, which were recognized within the cuticle of other avian species. The presence of the CL was confirmed by comparison of the shells with or without pre-treatment with potassium hydroxide (Figs. 1G, H).

\section{2) Light and transmission electron microscopy}

Light microscopic examination of semi-thin sections allowed characterization of the overall organic matrix architecture of the decalcified eggshells (Fig. 2A): the four major zones, SM, MZ, PR, and CL, of the eggshells were also recognized in the sections stained with toluidine blue, as already shown by SEM.

TEM examination of the SM identified the LM, about $2 \mu \mathrm{m}$ thick, and the fibrous elements in this zone (Figs. 2B, C). The LM appeared as a heterogeneous structure consisting of electron-dense and less electron-dense materials. The LM contacted with the fibers of the inner SM without forming any special structure between them (Fig. 2B). The fibers of the inner SM showed a round or ovoid contour in cross section and had a homogeneous content of moder- ately electron-dense material although they were associated with highly electron-dense particles (Fig. 2B). The fibers of the outer SM were thicker in diameter than those of the inner SM, but no essential difference was found in ultrastructure between them. In the apices of the mammillare, the fibers of the outer SM connected to the apices were surrounded by a mantle-like layer of less electron-dense material, from which fine fibrils projected mainly to the PR (Fig. 2C). These fibrils, $27.8 \mathrm{~nm}$ in mean diameter, were densely aggregated in the core portion of the mammillare, where they existed with occasional vesicles and electron-lucent flocculent material (Fig. 2D). The PR was characterized by the presence of numerous vesicles with an electron-dense fringe, $0.9 \mu \mathrm{m}$ in mean diameter, and embedded in a matrix substance together with the flocculent material (Fig. 2E). The vesicles and the flocculent material became remarkably less dense in the external part of the PR than in the major PR zone. The CL was composed of an electron-dense amorphous material, and some parts of it were associated with a thin layer of the fibrillar elements (Fig. 2F).

\section{3) X-ray compositional microanalysis}

The distribution patterns of $\mathrm{Ca}, \mathrm{Mg}$, and $\mathrm{P}$ in the radial face of the eggshell were simultaneously revealed (Fig. 3). The concentration (or count) of $\mathrm{Ca}$ was markedly high throughout the true shell (MZ and PR), but was low or almost negligible in the SMs. In the inner zone of the shell, the concentration curve of Ca tended to be slightly reduced. On the other hand, a locally high concentration of $\mathrm{Mg}$ was noted immediately beneath the inner surface of the shell, approximately corresponding to the MZ. The peak Mg concentration was found in the inner part occupying about $1 / 6$ (ca. 17\%) of the total thickness of the radial face of the true shell and consisted of two indistinct peaks. In the outer part of the shell and also throughout the shell membrane, the $\mathrm{Mg}$ concentration was clearly low. The concentration of $\mathrm{P}$ was slightly higher in the surface region, corresponding to the external zone (or surface crystal layer), than in any other layers of the shell or in the SMs and tended to decline progressively toward the inner zone.

\section{DISCUSSION}

The present study on the eggshell of the Blacktailed Gull demonstrated four major zones in the radial face: the shell membranes, the mammillary zone, 

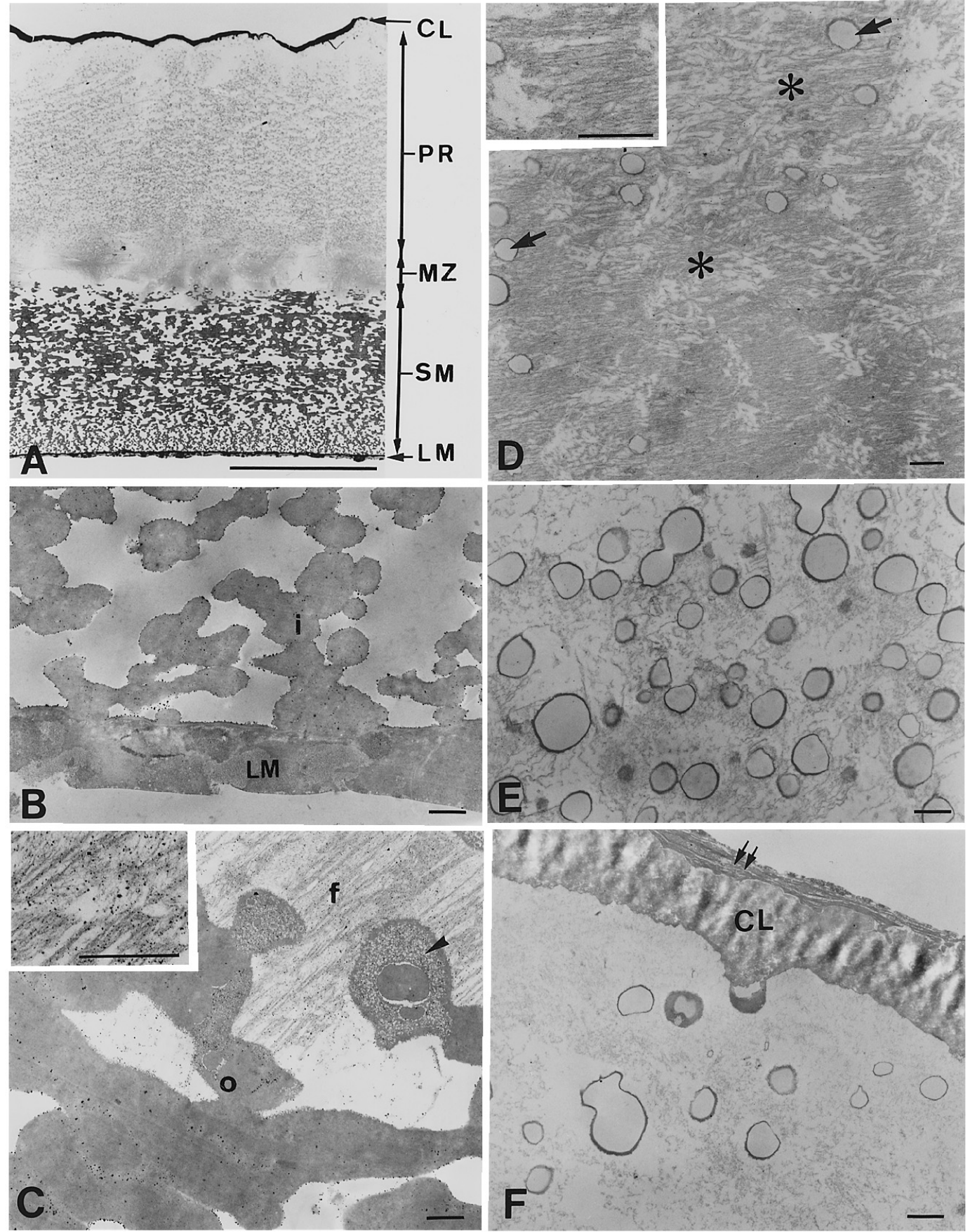

Fig. 2. Light (A) and TEM (B-F) micrographs of the decalcified eggshell. (A) cross section of full thickness of the eggshell showing the shell membrane (SM), mammillary layer (MZ), palisade region (PR), and cuticle layer (CL). (B) part of the SM showing the limiting membrane (LM) and inner SM (i). (C) part of the outer SM (o) showing fine fibrils (f) in the matrix and less electron dense mantle layer (arrowhead) of filaments. (D) core portion of the mammillare, characterized by dense aggregation of fine fibrils (asterisks) and a few vesicles (arrows). (E) part of PR of the true shell proper, showing a spongy feature depicted by numerous vesicles with an electrondense fringe. (F) the external part of the shell, showing a few vesicles in the matrix and the cuticle layer (CL) associated with discontinuously with a thin coat consisting of fibrillar material (double arrow). Insets (C and D) show the fine fibrils. Scale bar $=100 \mu \mathrm{m}(\mathrm{A}), 1 \mu \mathrm{m}(\mathrm{B}-\mathrm{F})$. 


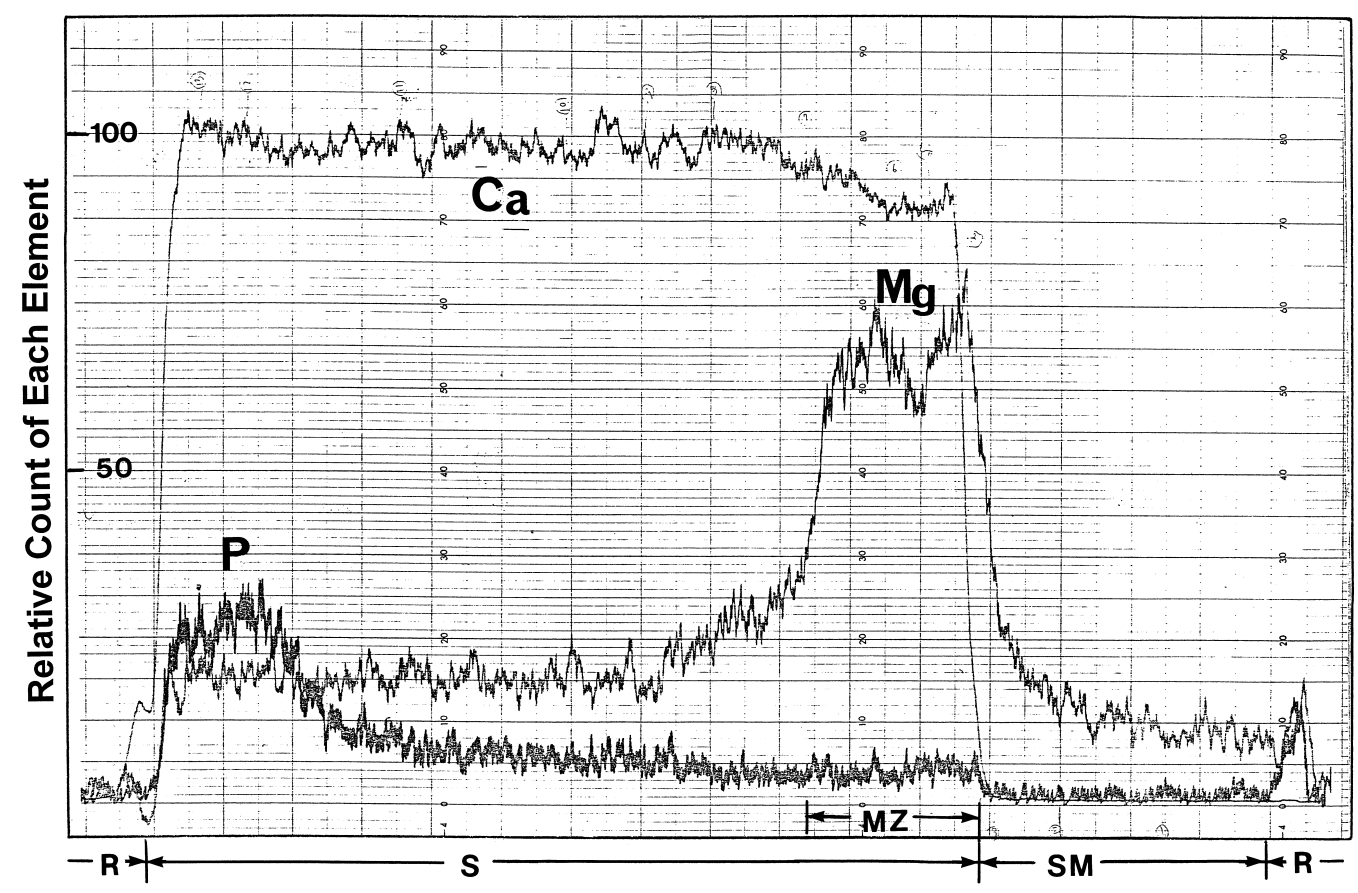

Fig. 3. Different patterns of $\mathrm{Ca}, \mathrm{Mg}$, and $\mathrm{P}$ concentration (counts) in the radial face of the eggshell obtained by $\mathrm{X}$-ray compositional microanalysis. MZ mammillary zone; R resin as embedding material, S true shell; SM shell membrane.

the palisade region, and the cuticle layer, as recognized in the hen eggshell (Dennis et al. 1996). According to Gilbert (1979), there are generally three parts to the eggshell; the shell membranes, the testa or calcified portion, and the cuticle. The shell membranes consist of the inner membrane (membranae testae interna) and the more complex outer membrane (membranae testae externa). Similarly, Tullet (1984) recognized three layers: the shell membranes, the true shell, and the cuticle. Furthermore, the limiting membrane as the third element of the shell membranes was distinguished between the inner shell membrane and the egg albumin. According to his view, the true shell includes three parts: the cone layer, the palisade layer, and the surface crystal layer. On the other hand, Mikhailov (1995a) applied similar or even identical terms, i.e. the shell membranes, the true eggshell, and the accessory material, to the gross structure of the eggshell. At the ultrastructural level, he recognized fiver layers in the eggshell: the basal plate groups, the radiating elements, the squamatic zone, the external zone, and the cuticle cover. In spite of minor differences in the usage or application of the terms, the present study prefers the view of Tullet (1984), for we identified the limiting membrane and demonstrated sublayers in the true shell proper, which correspond to the surface crystal layer and the cone layer (Tullet 1984).

TEM data on the organic parts of eggshells are few and exclusively concerned with the eggshell from the domestic fowl (Tan et al. 1992; Dennis et al. 1996; Fraser et al. 1998, 1999). The present TEM data were obtained from decalicified samples, not from intact and decalcified samples as in the domestic fowl (Dennis et al. 1996). Nevertheless, no essential difference was found in the fine structure of the shell membrane between the domestic fowl and the Black-tailed Gull. Concerning the structure and distribution of the vesicles embedded in the shell matrix, there were similarities and dissimilarities between the domestic fowl and the gull. In the gull, as in the domestic fowl, the vesicles were densely distributed in the palisade region, but scattered in the mammillary layer and the external part of the palisade region. Their size and fine structure, in particular the presence of the electron-dense fringe, were almost the same in the domestic fowl and the gull. However, the ordered stacking of co-aligned sheets of vesicles reported to exist in the domestic fowl was indistinct or almost invisible in the gull. Moreover, in the domestic fowl (Dennis et al. 1996), two types of vesicles were discerned based on their topography and internal structure, i.e. 
the palisade vesicles and the crown vesicles. The vesicles in the gull, with no relation to their topography in the shell, seem to be equivalent to the palisade vesicles in the domestic fowl. The biological significance of this difference remains unknown.

The microscopic features and chemical composition of the cuticle and/or cover layers of eggshells show a considerable variation among different taxonomic groups or species. In the domestic fowl, the cuticle has a vesicular structure (Simons \& Wierts 1966) and consists of protein, fat and polysaccharides (Tyler \& Simkiss 1959; Wedral et al. 1974). Such an organic cuticle was found in the eggshells of Guinea Fowl Numida meleagris, and the Greater Flamingo Phoenicopterus ruber (Tullet et al. 1976). However, among species belonging to the Gaviiformes, Pelecaniformes, and Podicipediformes, the external layer has a chalky appearance, is rich in inorganic material, and is more appropriately termed a cover rather than a cuticle (Tyler 1969; Tullet et al. 1976; Board et al. 1984). According to Tullet et al. (1976), this layer is rich in vaterite, a form of calcium carbonate. The vaterite-rich spherules in the eggshell cover of the grebes (Podicipidae) were investigated in details by $\mathrm{X}$-ray compositional microanalysis and shown to contain O, Ca, P, and S (Board et al. 1984). The vaterite-containing spherules were present also in the cuticle of the hen egg (Dennis et al. 1996). Thus, the external layer of the eggshell of the Black-tailed Gull appears to be rather simple and differs considerably from that of members of the Podicipediformes, Pelecaniformes, and Galliformes. The reason for (or background causing) such a difference is obscure, but various factors may be concerned. These include, for example, the physiology of the oviduct, the life-style of the birds, and their breeding strategies in association with the microenvironment of egg-laying sites.

The markedly high concentration of Ca throughout the true shell is not surprising, because it is generally known that the avian eggshell is a biomineralized composite ceramic consisting of calcium carbonate. The higher concentration of $\mathrm{Mg}$ in the cone layer is, however, of particular interest. It has been generally considered that the cones or mammillares are special parts, where calcification of the eggshell initiates and ultimately the porosity and thickness of the shell are determined (Tullet \& Board 1977; Tyler \& Fowler 1979). Previous studies have shown two peaks in the concentration of $\mathrm{Mg}$ in the eggshells of galliform birds, one in the cone layer and the other at the outer edge of the shell (Itoh \& Hatano 1964; Quintana \&
Sandoz 1978; Board \& Love 1980). In contrast, only one peak (in the cone layer) was found in the shells of many other birds (Board \& Love 1980). According to Board and Love (1980), the eggshells of charadriiform birds are of the latter type, being consistent with the present results on the Black-tailed Gull. The functional significance of $\mathrm{Mg}$ in eggshell formation is not yet clear (Board \& Love 1980), although its inhibitory role for nucleation or calcite growth during shell formation has been postulated (Bernar 1975).

\section{ACKNOWLEDGMENTS}

I am grateful to Dr. M. Nakayama, Department of Material Science of Nippon Dental University, for his technical support for the X-ray compositional microanalysis.

\section{REFERENCES}

Becking JH (1975) The ultrastructure of the avian eggshell. Ibis 117: 143-151.

Bellairs R \& Boyde A (1969) Scanning electron microscopy of the shell membranes of the hen's egg. Z Zellforsch Mikrosk Anat 96: 237-249.

Bernar RA (1975) The role of magnesium in crystal growth of calcite and aragonite from sea water. Geochim Cosmochimi Acta 39: 489-504.

Board RG \& Love G (1980) Magnesium distribution in avian eggshells. Comp Biochem Physiol 66A: 667-672.

Board RG, Perrott HR, Love G \& Scott VD (1984) The phosphate-rich cover of the eggshells of grebes. J Zool (Lond) 203: 329-343.

Dennis JE, Xiao S-Q, Agarwal M, Fink DJ, Heuer AH \& Caplan AL (1996) Microstructure of matrix and mineral components of eggshells from White Leghorn chickens (Gallus gallus). J Morphol 228: 287-306.

Fraser AC, Bain MM \& Solomon SE (1998) Organic matrix morphology and distribution in the palisade layer of eggshells sampled at selected periods during lay. Br Poult Sci 39: 225-228.

Fraser AC, Bain MM \& Solomon SE (1999) Transmission electron microscopy of the vertical crystal layer and cuticle of the eggshell of the domestic fowl. $\mathrm{Br}$ Poult Sci 40: 626-631.

Fujii S \& Tamura T (1969) Scanning electron microscopy of the hen's egg shell. J Fac Fish Anim Husb Hiroshima Univ 8: 95-98.

Gilbert AB (1979) Female genital organs. In King AS \& Mclelland J (eds) Form and function in birds. Vol 1. pp 237-360. Academic Press, London.

Itoh H \& Hatano T (1964) Variation of magnesium and 
phosphorus deposition rates during eggshell formation. Poul Sci 43: 65-80.

Mikhailov KE (1995a) Eggshell structure in the shoebill and pelecaniform birds: comparison with Hamerkop, herons, ibises, and storks. Can J Zool 73: 1754-1770.

Mikhailov KE (1995b) The evolutionary implications of eggshell structure in falconiform and ciconiiform birds. Cour Forsch Senckenberg 181: 337-355.

Paganelli CV, Ar A, Rahn H \& Wangensteen OD (1975) Diffusion in the gas phase: the effect of ambient pressure and gas composition. Resp Physiol 25: 247-258.

Quintana C \& Sandoz D (1978) Coquille de l'oeuf de caille: étude ultrastructurale et cristallographique. Calcif Tiss Res 25: 145-159.

Rahn H \& Paganelli CV (1981) Gas exchange in avian eggs. Buffalo Press, New York.

Richards PD, Richards PA \& Lee ME (2000) Ultrastructural characteristics of ostrich eggshell: outer shell membrane and the calcified layers. J S Afr Vet Assoc 71: 97-102.

Romanoff AL \& Romanoff AJ (1949) The avian egg. John Wiley and Sons Inc, New York.

Simons PCM \& Wierts G (1966) The ultrastructure of the surface of the cuticle of the hen egg in relation to egg cleaning. Poult Sci 45: 1153-1162.

Tan CK, Chen TW, Chan HL \& Ng LS (1992) A scanning and transmission electron microscopic study of the membranes of chicken egg. Histol Histopathol 7: 339-345.

Tullet SG (1984) The porosity of avian eggshells.
Comp. Biochem Physiol 78A: 5-13.

Tullet SG \& Board RG (1977) Determinants of avian eggshell porosity. J Zool (Lond) 183: 203-211.

Tullet SG, Board RG, Love G, Perrott HR \& Scott VD (1976) Vaterite deposition during eggshell formation in the cormorant, gannet and shag, and in "shell-less" eggs of the domestic fowl. Acta Zool (Stockh) 57: 79-87.

Tung MA \& Richard JH (1972) Ultrastructure of the hen's egg shell membrane by electron microscopy. J Food Sci 37: 277-281.

Tyler C (1964) A study of the egg shells of the Anatidae. Proc Zool Soc Lond 142: 547-583.

Tyler C (1965) A study of the egg shells of the Sphenisciformes. J Zool (Lond) 147: 1-19.

Tyler C (1966) A study of the egg shell of the Falconiformes. J Zool (Lond) 150: 413-425.

Tyler C (1969) A study of the egg shells of the Gaviiformes. J Zool (Lond) 158: 395-412.

Tyler C \& Fowler S (1979) The size, shape and orientation of pore grooves in the egg shells of Rhea sp. J Zool (Lond) 187: 283-290.

Tyler C \& Simkiss K (1959) Studies on egg shells XII. Some changes in the shell during incubation. J Sci Food Agr 10: 611-615.

Wedral EM, Vadehra DV \& Baker RC (1974) Chemical composition of the cuticle and the inner and outer shell membrane from egg of Gallus gallus. Com Biochem Biophys 47B: 631-640. 